\title{
Quantum dots for multimodal molecular imaging of angiogenesis
}

\author{
Willem J. M. Mulder · Gustav J. Strijkers • \\ Klaas Nicolay $\cdot$ Arjan W. Griffioen
}

Received: 6 June 2010/ Accepted: 7 June 2010/Published online: 16 June 2010

(c) The Author(s) 2010. This article is published with open access at Springerlink.com

\begin{abstract}
Quantum dots exhibit unique optical properties for bioimaging purposes. We have previously developed quantum dots with a paramagnetic and functionalized coating and have shown their potential for molecular imaging purposes. In the current mini-review we summarize the synthesis procedure, the in vitro testing and, importantly, the in vivo application for multimodal molecular imaging of tumor angiogenesis.
\end{abstract}

Keywords Quantum dots - Molecular imaging ·

Angiogenesis - Multimodal imaging - Magnetic resonance imaging · Fluorescence imaging · Intravital microscopy

\section{Introduction}

Quantum dots (QDs), semiconductor nanocrystals, have exceptional optical properties, which make them ideally suited for a number of applications in biomedical imaging, with several important advantages over fluorescent dyemolecules $[1,2]$. The absorption spectrum of a QD is very

W. J. M. Mulder ( $\square)$

Translational and Molecular Imaging Institute,

Mount Sinai School of Medicine, One Gustave L. Levy Place,

Box 1234, New York, NY 10029, USA

e-mail: willem.mulder@mountsinai.org

G. J. Strijkers · K. Nicolay

Biomedical NMR, Department of Biomedical Engineering, Eindhoven University of Technology, Eindhoven, The Netherlands

\section{A. W. Griffioen}

Angiogenesis Laboratory, Department of Medical Oncology, VUmc-Cancer Center Amsterdam, VU University Medical Center, Amsterdam, The Netherlands broad, while the emission spectrum is narrow. The exact optical properties of QDs can be tuned by a precise control of composition and size, allowing the synthesis of QDs with emission wavelengths spanning from the near UV to near infrared (NIR), making QDs particularly suitable for multiplexed imaging [3]. Importantly for biological applications, the surface of QDs can be easily modified to render them water-soluble and biocompatible as well as to allow the introduction of additional functionalities and to make them target-specific. Dubertret and colleagues were the first to apply quantum dots to a living organism (Xenopus embryos) for in vivo imaging purposes [4]. To that end they encapsulated the QD nanocrystals in phospholipid micelles. Previously, we modified their procedure to create QD-based nanoparticles that can be detected by optical techniques as well as magnetic resonance imaging (MRI). Using this approach we have created QD based nanoparticles that specifically bind activated endothelial cells to image ongoing angiogenesis $[5,6]$.

\section{Synthesis of paramagnetic QD-micelles}

The original procedure involves mixing of QDs that are coated with hydrophobic capping molecules with an excess of PEGylated phospholipids (PEG-lipids) and Gd-DTPA labeled lipids (Gd-DTPA-DSA) in chloroform. Upon evaporation of the solvent, a mixed film containing the lipids and QDs is formed. Hydrating and heating this film results in the formation of paramagnetic micelles that contain a QD in their core. This procedure results in the formation of a relatively large fraction of aggregated QDs coated by the PEG-lipids. To increase the yield of paramagnetic micelles with a single QD core we modified the procedure as illustrated in Fig. 1. The new method involves 
Fig. 1 Schematic representation of the synthesis of QDs with a paramagnetic micellar coating. QDs and lipids in chloroform are slowly infused in hot water that, via chloroform-in-water emulsions, swiftly form micelles when chloroform evaporates, some of which have a QD core
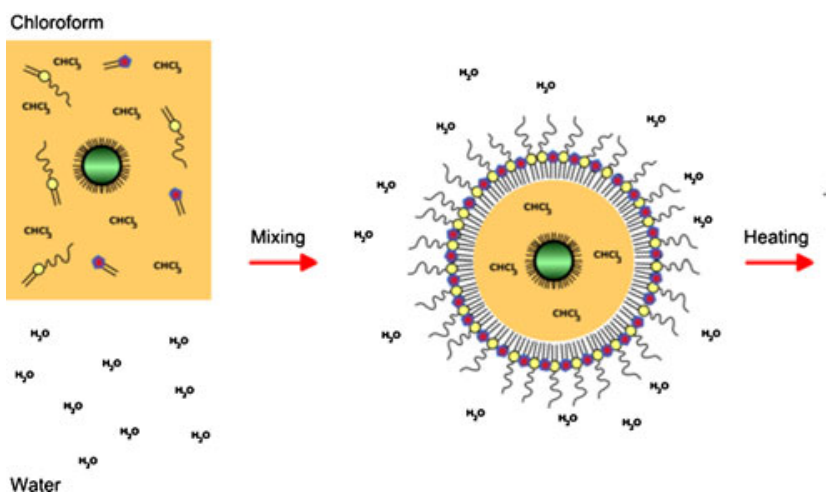

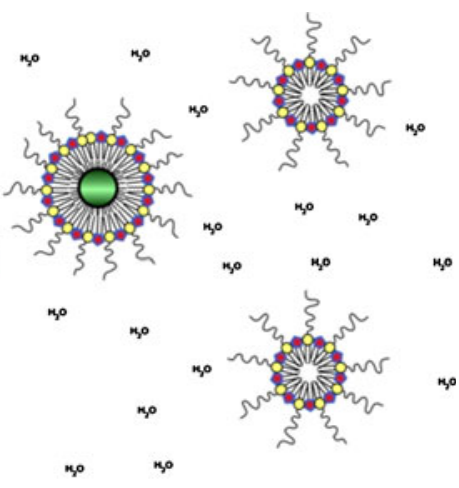

slow infusion of the chloroform QD/lipid mixture into hot water, which results in the formation of chloroform-inwater emulsions that swiftly form micelles as chloroform evaporates. The resulting mix of QD core micelles and empty micelles is separated by centrifugation methods. The relaxivity of purified paramagnetic QD-micelles is approximately $2,000 \mathrm{mM}^{-1} \mathrm{~s}^{-1}$ (per $\mathrm{mM}$ nanoparticles) at a clinically relevant field strength of $1.41 \mathrm{~T}$, which makes them attractive for molecular MRI purposes [7, 8].

By the inclusion of maleimide functionalized PEG-lipids (Mal-PEG-lipid), the paramagnetic QD-micelles can be functionalized via a sulfhydryl-maleimide coupling method. Using this strategy, we have shown the conjugation of $\alpha v \beta 3$-integrin specific cyclic RGD peptides [5,9] and phosphatidylserine specific Annexin A5 proteins [6] to the paramagnetic QD-micelles, but also the conjugation of E-selectin specific antibodies to paramagnetic liposomes [10].

\section{In vitro targeting and imaging}

To assess the biological specificity of the RGD and Annexin A5 conjugated paramagnetic QD-micelles (abbreviated as RGD-pQD and A5-pQD, respectively) human umbilical vein endothelial cells (HUVEC) and Jurkat cells were used for in vitro testing, respectively. Growth factor activated and proliferating HUVEC overexpress the $\alpha \mathrm{v} \beta 3$-integrin, while Jurkat cells exposed to anti-Fas become apoptotic and expose the negatively charged phospholipid phosphatidylserine (PS) at the outer layer of the cell membrane. Both markers are known to be expressed in angiogenic blood vessels. In Fig. 2a a $\mathrm{T}_{1}$-weighted $\mathrm{MR}$ image of loosely packed cells, 1.5 million per pellet, is shown. The pellet of cells incubated with the targeted RGD-pQDs appeared much brighter than those that were incubated with non-targeted pQDs or that were not incubated with contrast agent. A fluorescence microscopy image of HUVEC that had been incubated with green emitting RGD-pQDs is depicted in
Fig. 2a (right) and showed the nanoparticles to be internalized, at a perinuclear location (inset). In Fig. 2b Jurkat cell pellets are shown that were illuminated with $254 \mathrm{~nm}$ UV light. The green emitting cell pellet originating from the A5pQDs incubated PS expressing cells can be clearly distinguished from the control cell pellets.

\section{Multimodality molecular imaging of tumor angiogenesis}

To evaluate the in vivo potential of RGD-pQDs we designed a study where different groups of tumor bearing mice were imaged in vivo with fluorescence intravital microscopy (IVM), MRI, or whole body fluorescence imaging (Fig. 3, left) [9]. IVM was used to monitor nanoparticle binding to the tumor vasculature in real-time. We observed labeling of tumor blood vessels within 5-10 min after the administration of RGD-pQDS. A typical fluorescence image is depicted in Fig. 3. Labeling of endothelial cells in the tumor vasculature by RGD-pQDs was found as far as $1 \mathrm{~cm}$ from the tumor periphery, indicative for a widespread activation of the blood vessels in close proximity of cancerous tissue. No endothelial cell labeling of blood vessels in the hind limb of the animals and of blood vessels in the ears of the animals was observed.

MRI was performed on a different group of animals that were intravenously injected with RGD-pQD. A high resolution $\mathrm{T}_{1}$-weighted image, acquired $45 \mathrm{~min}$ after contrast agent injection, revealed angiogenic activity to be mainly found at the rim of the tumor, which corresponds with the regions of the tumor with highest angiogenic activity.

Lastly, whole body fluorescence imaging was employed to visualize angiogenesis in nude mice that grew a tumor in their kidney (Fig. 3, bottom). This technique has a higher sensitivity and temporal resolution than MRI and thus allows faster screening of nanoparticle targeting, albeit 



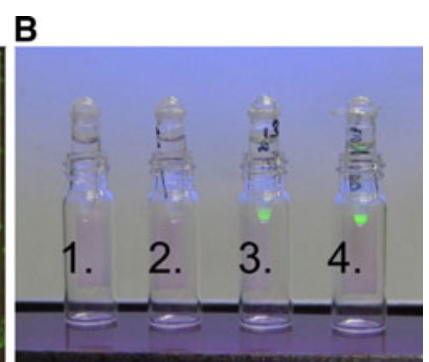

Fig. 2 In vitro targeting and imaging with QD-micelles. a, left $\mathrm{T}_{1}$-weighted MRI image of cells that were incubated with RGDpQDs, pQDS, or without contrast agent. a, right Fluorescence microscopy of HUVEC incubated with RGD-pQDs. b Pellets of Jurkatt cells of different incubations illuminated with $254 \mathrm{~nm}$ UV light. From left to right: [1] Control cells. [2] Apoptotic cells treated with EDTA to remove $\mathrm{Ca}^{2+}$, which prevents binding [3] vital cells, and [4] apoptotic cells, all incubated with Annexin A5 functionalized QD-micelles revealed the highest uptake in situation [4]
Intravital fluorescence microscopy
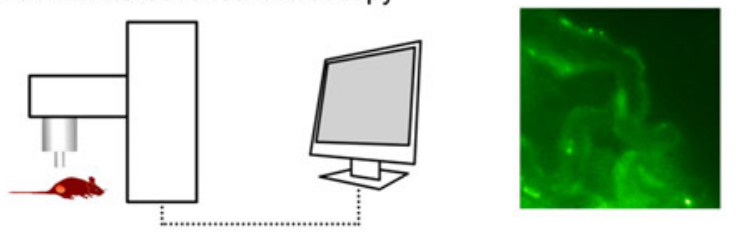

In vivo MRI


Fluorescence imaging


Fig. 3 Multimodal molecular imaging of tumor angiogenesis using QD-micelles. (Top) Intravital microscopy of microvessels in tumorbearing mice after intravenous injection of $\alpha \mathrm{v} \beta 3$-specific and paramagnetic QD-micelles. (Middle) $\mathrm{T}_{1}$-weighted MR images $45 \mathrm{~min}$ after the injection of the nanoparticles in a mouse bearing a subcutaneous tumor. The colored pixels in the tumor highlight the location of the MR-visible QD-micelles, predominantly in the rim of the tumor. (Bottom) Fluorescence image of a mouse, inoculated with a tumor in the kidney, following intravenous administration

with less spatial information than is the case for MRI. Altogether, IVM, MRI and fluorescence imaging are complementary imaging modalities that, when combined with our nanoparticle, can provide information about angiogenesis at the microscopic level of superficial blood vessels (IVM), at the whole mouse level with high sensitivity and temporal resolution (fluorescence imaging), as well as three dimensional information of the inside of the mouse and tumor (MRI).

\section{Discussion and conclusion}

QDs represent an excellent tool for multimodal molecular imaging of angiogenesis. It was recently demonstrated that these nanocrystals can be used to image the tumor microenvironment and that differently sized quantum dots can be used to assess the permeability of the tumor microvasculature [11]. Subsequent to the lipid-based QD platform we introduced, Oostendorp and colleagues demonstrated the functionalization of QDs with paramagnetic dendritic wedges and CD13 specific cNGR peptides to allow MR molecular imaging of tumor angiogenesis [12] as well as angiogenesis after myocardial infarction [13]. The fluorescence properties of the QDs were exploited to validate their findings with ex vivo two-photon laser scanning microscopy, which showed the QDs to be co-localized with endothelial cells. In addition to labeling QDs for MRI, studies have shown the value of the combination with positron emission tomography (PET) imaging. Cai and colleagues used radiolabeled and RGD peptide functionalized QDs to accomplish molecular imaging of tumor angiogenesis [14], while Chen et al. demonstrated the same using radiolabeled QDs that were functionalized with vascular endothelial growth factor [15]. Since our nanoparticle platform contains DTPA functionalized lipids the inclusion of a radiolabel should be relatively straightforward and may be introduced as a third label to additionally allow PET imaging. Interestingly, the flexibility of the QDmicelle platform also allows the possibility to include therapeutics [16] or additional targeting moieties to enhance the specificity of targeting, as was recently demonstrated by Kluza et al. for a liposomal nanoparticle [17].

In conclusion this mini-review presented the development of paramagnetic QD-micelles for MR and optical based molecular imaging. The ease of preparation and flexibility, as well as their use as a scaffold that is representative of other nanocrystals make them fruitful tools for biomedical imaging purposes. Their application may be 
extended to other imaging modalities, to other disease processes than angiogenesis, as well as for drug targeting purposes.

Acknowledgements The reported QD research was funded in part by the BSIK program entitled Molecular Imaging of Ischemic Heart Disease (project number BSIK03033) and the European Community EC-FP6-project Diagnostic Molecular Imaging (grant number LSHBCT-2005- 512146)

Open Access This article is distributed under the terms of the Creative Commons Attribution Noncommercial License which permits any noncommercial use, distribution, and reproduction in any medium, provided the original author(s) and source are credited.

\section{References}

1. Medintz IL, Uyeda HT, Goldman ER, Mattoussi H (2005) Quantum dot bioconjugates for imaging, labelling and sensing. Nat Mater 4:435-446

2. Michalet X, Pinaud FF, Bentolila LA et al (2005) Quantum dots for live cells, in vivo imaging, and diagnostics. Science 307: 538-544

3. Jaiswal JK, Mattoussi H, Mauro JM, Simon SM (2003) Longterm multiple color imaging of live cells using quantum dot bioconjugates. Nat Biotechnol 21:47-51

4. Dubertret B, Skourides P, Norris DJ et al (2002) In vivo imaging of quantum dots encapsulated in phospholipid micelles. Science 298:1759-1762

5. Mulder WJ, Koole R, Brandwijk RJ et al (2006) Quantum dots with a paramagnetic coating as a bimodal molecular imaging probe. Nano Lett 6:1-6

6. van Tilborg GA, Mulder WJ, Chin PT et al (2006) Annexin A5conjugated quantum dots with a paramagnetic lipidic coating for the multimodal detection of apoptotic cells. Bioconjug Chem 17:865-868

7. Weissleder R, Mahmood U (2001) Molecular imaging. Radiology 219:316-333

8. Mulder WJ, Strijkers GJ, van Tilborg GA, Griffioen AW, Nicolay K (2006) Lipid-based nanoparticles for contrast-enhanced MRI and molecular imaging. NMR Biomed 19:142-164

9. Mulder WJ, Castermans K, van Beijnum JR et al (2009) Molecular imaging of tumor angiogenesis using alphavbeta3integrin targeted multimodal quantum dots. Angiogenesis 12: $17-24$

10. Mulder WJ, Strijkers GJ, Griffioen AW et al (2004) A liposomal system for contrast-enhanced magnetic resonance imaging of molecular targets. Bioconjug Chem 15:799-806

11. Stroh M, Zimmer JP, Duda DG et al (2005) Quantum dots spectrally distinguish multiple species within the tumor milieu in vivo. Nat Med 11:678-682

12. Oostendorp M, Douma K, Hackeng TM et al (2008) Quantitative molecular magnetic resonance imaging of tumor angiogenesis using cNGR-labeled paramagnetic quantum dots. Cancer Res 68:7676-7683

13. Oostendorp M, Douma K, Wagenaar A et al (2010) Molecular magnetic resonance imaging of myocardial angiogenesis after acute myocardial infarction. Circulation 121:775-783

14. Cai W, Chen K, Li ZB, Gambhir SS, Chen X (2007) Dualfunction probe for PET and near-infrared fluorescence imaging of tumor vasculature. J Nucl Med 48:1862-1870

15. Chen K, Li ZB, Wang H, Cai W, Chen X (2008) Dual-modality optical and positron emission tomography imaging of vascular endothelial growth factor receptor on tumor vasculature using quantum dots. Eur J Nucl Med Mol Imag 35:2235-2244

16. Lukyanov AN, Torchilin VP (2004) Micelles from lipid derivatives of water-soluble polymers as delivery systems for poorly soluble drugs. Adv Drug Deliv Rev 56:1273-1289

17. Kluza E, van der Schaft DW, Hautvast PA et al (2010) Synergistic targeting of alphavbeta3 integrin and galectin-1 with heteromultivalent paramagnetic liposomes for combined MR imaging and treatment of angiogenesis. Nano Lett 10:52-58 